\title{
European survey of diagnosis and management of the polycystic ovary syndrome: results of the ESE PCOS Special Interest Group's Questionnaire
}

\author{
Gerard Conway, Didier Dewailly ${ }^{1}$, Evanthia Diamanti-Kandarakis ${ }^{2}$, \\ Hector F Escobar-Morreale ${ }^{3}$, Steven Franks ${ }^{4}$, Alessandra Gambineri ${ }^{5}$, \\ Fahrettin Kelestimur ${ }^{6}$, Djuro Macut ${ }^{7}$, Dragan Micic ${ }^{7}$, Renato Pasquali ${ }^{5,+}$, \\ Marija Pfeifer ${ }^{8}$, Duarte Pignatelli ${ }^{9}$, Michel Pugeat ${ }^{10}$, Bulent 0 Yildiz ${ }^{11}$ \\ on behalf of the ESE PCOS Special interest Group
}

Department of Endocrinology, University College London Hospitals, London NW1 2BU, UK, ${ }^{1}$ Department of Endocrine Gynaecology and Reproductive Medicine, Centre Hospitalier de Lille, Hopital Jeanne de Fiandre, Lille, France, ${ }^{2}$ Third Department of Medicine, Neuroendocrine Clinic, University of Athens-Medical School, Athens, Greece, ${ }^{3}$ Department of Endocrinology and Nutrition, Universidad de Alcalá and Hospital Universitario Ramón y Cajal and Centro de Investigación Biomédica en Red Diabetes y Enfermedades Metabólicas Asociadas CIBERDEM and Instituto Ramón y Cajal de Investigación Sanitaria IRYCIS, Madrid, Spain, ${ }^{4}$ Imperial College London, Institute of Reproductive and Developmental Biology, London, UK, ${ }^{5}$ Division of Endocrinology, Department of Medical and Surgical Sciences, St Orsola-Malpighi Hospital, University Alma Mater Studiorum, Via Massarenti 9, 40138 Bologna, Italy, ${ }^{6}$ Department of Endocrinology, School of Medicine, Erciyes University, Kayseri, Turkey, ${ }^{7}$ Clinic for Endocrinology, Diabetes and Metabolic Diseases, School of Medicine, University of Belgrade, Belgrade, Serbia, ${ }^{8}$ Department of Endocrinology, Diabetes and Metabolic Diseases, University Medical Centre, Ljubljana, Slovenia, ${ }^{9}$ Department of Endocrinology, Hospital S. Joao, Porto, Portugal, ${ }^{10}$ Inserm U863 IFR6, Fédération d'Endocrinologie, Groupement Hospitalier Est, Hospices Civils de Lyon, Université Lyon-1, Lyon, France and ${ }^{11}$ Division of Endocrinology and Metabolism, Department of Internal Medicine, Hacettepe University School of Medicine, Ankara, Turkey

${ }^{\dagger}$ Group coordinator

Correspondence should be addressed to R Pasquali

Email renato.pasquali@unibo.it

\begin{abstract}
Background: There is evidence for differences between endocrinologists and other specialists in their approach to diagnosis and management of the polycystic ovary syndrome (PCOS).

Objective: A mailed survey consisting of a simple questionnaire aiming to understand current practice for diagnosis and management of the PCOS by specialists across Europe.

Methods: The questionnaire consisted of 23 questions grouped to achieve information on i) the general characteristics of the respondents, ii) patients with PCOS seen by endocrinologists, iii) the main diagnostic criteria, iv) biochemical parameters used in the differential diagnosis of hyperandrogenism, v) long-term concerns, and, finally vi) treatment choices. A total of 357 questionnaires representing $13.3 \%$ of the members of European Society of Endocrinology (ESE) were available for final analysis; $93 \%$ of the respondents were endocrinologists

Results: In relation to the diagnostic criteria, respondents were most likely to select menstrual irregularity as the most frequent criteria used for the diagnosis of PCOS although very high rates were achieved for the use of hirsutism and biochemical hyperandrogenism. It therefore appears that the NIH criteria were followed by the majority of respondents. The most frequent biochemical parameters in the differential diagnosis of hyperandrogenism were total testosterone or free androgen index. Obesity and type 2 diabetes were regarded as the principal long-term concerns for PCOS. The most common treatments for patients with PCOS were metformin (33\%), lifestyle modification (25\%), and oral contraceptives (22\%). More direct treatments of infertility include clomiphene citrate alone or in combination with metformin, prescribed by 9 and $23 \%$, respectively, whereas only $6 \%$ used other methods for induction of ovulation.
\end{abstract}

(C) 2014 European Society of Endocrinology Printed in Great Britain
Published by Bioscientifica Ltd. 
Conclusion: The survey produced by ESE is a good start for evaluating the perspective in the diagnosis and treatment of PCOS by endocrinologists in Europe.

\section{Introduction}

The requirement that all pathological conditions characterized by androgen excess in women should attract increasing interest from endocrinologists is shared by many members of the European Society of Endocrinology (ESE). The polycystic ovary syndrome (PCOS) represents the most common clinical condition characterized by androgen excess, but the diagnosis can be made only after the exclusion of all other potential conditions with a welldefined etiology $(1,2,3)$. Therefore, androgen excess, clinically indicated by hirsutism and established by the finding of increased blood testosterone level, is usually considered as the main criterion for the definition of PCOS by endocrinologists. In contrast, menses abnormalities and chronic anovulation together with polycystic ovarian morphology in ultrasound are the criteria favored by gynecologists, whose primary interest is usually focused on ovarian dysfunction (4). Both approaches have been a matter of discussion with some controversies that preclude a clear and decisive consensus on management $(1,2,3)$. It should be noted that none of the diagnostic criteria for PCOS $(1,3)$ are the result of a formal consensus process, in fact they were based on expert opinion rather than evidence. This may justify some different perspectives and concerns on the major criteria currently used to define PCOS.

However, even in women whose primary reason for presentation is ovarian dysfunction, PCOS is often characterized by the presence of different degrees of insulin resistance and compensatory insulin excess, and many patients are overweight or obese, conditions which play an important role in the pathogenesis of androgen excess (4). In addition, women with PCOS are characterized by an increased susceptibility to develop glucose intolerance and type 2 diabetes (T2D) $(5,6)$. These metabolic disorders are particularly common in women with the classic hyperandrogenic-anovulatory phenotype $(7,8)$. Arguments in favor of the inclusion of metabolic issues while characterizing the PCOS phenotype in each single patient have emerged in recent years, although this is still a matter of intense debate $(7,8)$. Some different perspectives in the definition, diagnosis, and treatment of
PCOS between endocrinologists and gynecologists have been documented (9). As an unfavorable consequence, women might receive a different diagnosis or treatment depending on the specialist to whom they are referred.

We therefore planned a survey, on behalf of the ESE, consisting of a simple questionnaire aimed at understanding the usual procedure in diagnosis and management of PCOS by specialists, mostly endocrinologists, across Europe. It is of importance to note that the methodology we employed in the current study is very similar to that pursued by the former NIH criteria (1) that was based on a survey of the participants in the NIHNICHD conference on PCOS.

\section{Materials and methods}

In 2012, the ESE PCOS Special Interest Group prepared a questionnaire to be sent to all ESE members, with the aim of collecting some information on the current practice in the management of PCOS in different European countries as a first step toward preparing a position statement on the management of this condition. The study was approved by the institutional review board of the ESE.

The questionnaire consisted of 23 questions, some of which envisaged two or more responses. Respondents were characterized according to age, type of hospital or medical school, clinical speciality, and practice settings. In addition, specific questions were prepared to obtain information on i) the estimated prevalence of PCOS in the country of residence, ii) the diagnostic criteria used to define PCOS, iii) treatment choices, iv) long-term concerns about PCOS, and v) collaboration with other specialists. The list of questions was developed on the basis of scientific evidence on which all the members of the group agreed at the first meeting in Paris, January 2012. Conversely, several other questions were defined in order to obtain information from members of the ESE on their most common diagnostic procedures and on their concerns regarding metabolic issues. 
Table 1 Information and questions of the questionnaire (list of the answers in brackets).

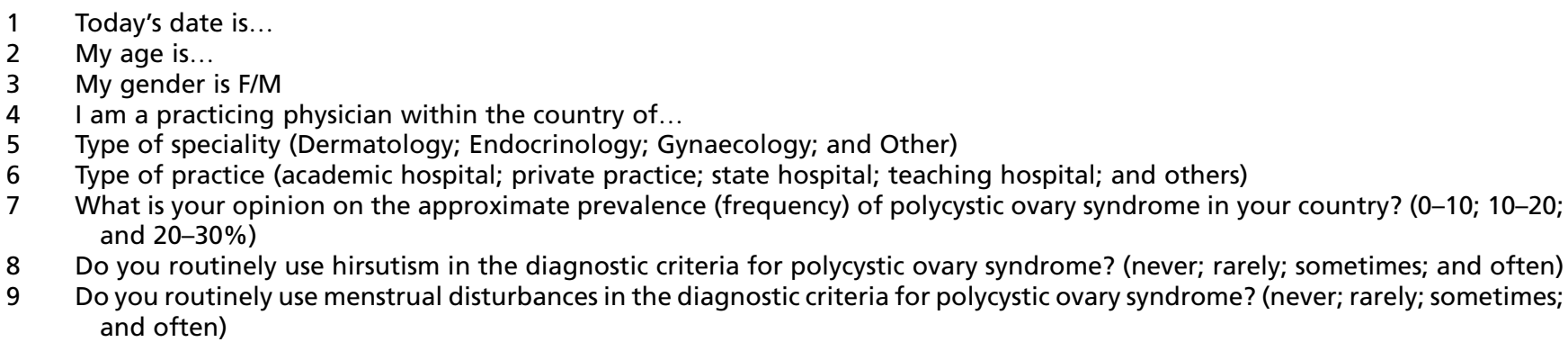

8 Do you routinely use hirsutism in the diagnostic criteria for polycystic ovary syndrome? (never; rarely; sometimes; and often)

9 Do you routinely use menstrual disturbances in the diagnostic criteria for polycystic ovary syndrome? (never; rarely; sometimes; and often)

10 Do you routinely use biochemical hyperandrogenism in the diagnostic criteria for polycystic ovary syndrome? (never; rarely; sometimes; and often)

11 Do you routinely use pelvic ultrasonography in the diagnostic criteria for polycystic ovary syndrome? (never; rarely; sometimes; and often)

12 Do you routinely use anti-Müllerian hormone for the diagnosis of polycystic ovary syndrome? (never; rarely; sometimes; and often)

13 Do you ask your patients about family history of diabetes, cardiovascular disease, and polycystic ovary syndrome? (yes, no)

14 Of those patients that you have diagnosed with polycystic ovary syndrome, what was the most common reason for clinic attendance? (hirsutism, infertility, menstrual disturbances, metabolic disorders, and obesity)

15 Which are the two most common biochemical parameters you use in the differential diagnosis of hyperandrogenism in your patients? (androstenedione, DHEAS, total testosterone, free testosterone (direct measurement), FAl, 17OH progesterone, and prolactin)

16 How many patients with polycystic ovary syndrome have you seen in the last year? $(<50 ; 50-200 ;$ and $>200)$

17 What is the most frequent age of diagnosis of polycystic ovary syndrome in your practice? $(<20 ; 20-30 ;$ and $>30)$

18 What is the most frequent source of patients with polycystic ovary syndrome in your practice? (dermatologist; endocrinologist; gynaecologist; general practitioner; self-referral; ultrasound specialist/practitioner; and other (please specify))

19 Do you run a multidisciplinary service for patients with polycystic ovary syndrome? (yes, no)

20 Which specialist is collaborating with you for the management of patients with polycystic ovary syndrome? (cardiologist; dermatologist; endocrinologist; gynaecologist; nutritionist; psychologist; and other (please specify))

21 What is your most important long-term concern on polycystic ovary syndrome? (please tick one box) (infertility; cardiovascular diseases; obesity and type 2 diabetes; endometrial cancer; and psyco-social problems)

22 Which treatments do you most commonly prescribe for most of your patients with polycystic ovary syndrome? (antiandrogens; laser depilation; lifestyle modifications; metformin; and oral contraceptives)

23 Which treatments do you most commonly prescribe for most of your patients with polycystic ovary syndrome regarding fertility? (please tick the treatments most frequently used) (clomiphene citrate; clomiphene citrate + metformin; lifestyle modifications; metformin; and ovulation inductors)

The complete list of questions and of all potential answers is reported in Table 1. In June 2012, the ESE secretariat placed the questionnaire on the ESE website and the completed questionnaires were collected between June 2012 and April 2013. Every member of the ESE received an email invitation sent on two occasions during this period.

Only 135 questionnaires (5\%) were obtained using the web-based survey (http://www.bioscientifica.com) organized by Bioscientifica Ltd (Bristol, UK). In order to achieve the goal, a further effort was planned during the 15th European Congress of Endocrinology held in Copenhagen, Denmark, 27th April-1st May 2013, when the collection of questionnaires was considered completed. At the end, a total of 364 questionnaires (corresponding to $13.5 \%$ of ESE members) were available for analysis, for a $4.8 \%$ error at the $95 \%$ confidence level. No duplicates were found.

\section{Statistical analysis}

Descriptive statistics and Student's $t$-test or Fisher's exact test were used to analyze the data, as needed. A level of $P<0.05$ was considered significant. Data were analyzed by SPSS Software version 13.0 (SPSS, Inc.).

\section{Results}

\section{General characteristics of the respondents}

Of the completed questionnaires, seven (1.9\%) were eliminated because the respondents did not reply to at 
Table 2 Characteristics of the members of the ESE who completed the survey.

\begin{tabular}{|c|c|c|}
\hline & $\begin{array}{c}\text { Numbers } \\
(n=357)\end{array}$ & $\begin{array}{c}\text { Percentage } \\
(\%)\end{array}$ \\
\hline \multicolumn{3}{|l|}{ Age } \\
\hline \multicolumn{3}{|l|}{$48.8 \pm 11.2$ (range $24-80$ years) } \\
\hline \multicolumn{3}{|l|}{ Gender } \\
\hline Males & 152 & 42.6 \\
\hline Females & 201 & 56.3 \\
\hline ND & 4 & 1.1 \\
\hline \multicolumn{3}{|l|}{ Countries $^{a}$} \\
\hline European & 268 & 75.1 \\
\hline Not European & 74 & 20.7 \\
\hline Unspecified & 15 & 4.2 \\
\hline \multicolumn{3}{|l|}{ Type of practice } \\
\hline Academic hospital & 145 & 40.6 \\
\hline State hospital & 73 & 20.4 \\
\hline Teaching hospital & 65 & 18.2 \\
\hline Private practice & 70 & 19.6 \\
\hline Others & 4 & 1.1 \\
\hline \multicolumn{3}{|l|}{ Speciality } \\
\hline Endocrinology & 332 & 92.9 \\
\hline Gynaecology & 13 & 3.6 \\
\hline $\begin{array}{l}\text { Other (internal medicine, } \\
\text { general practitioner, pediatric, } \\
\text { diabetology, and research) }\end{array}$ & 12 & 3.4 \\
\hline
\end{tabular}

${ }^{a}$ Countries of the respondents were Albania, Algeria, Australia, Austria, Bahrain, Belgium, Bosnia and Herzegovina, Brazil, Bulgaria, Canada, Colombia, Croatia, Cyprus, Czech Republic, Denmark, Ecuador, Egypt, Estonia, Finland, France, Georgia, Germany, Greece, Hungary, Iceland, India, Iran, Ireland, Italy, Japan, Jordan, Latvia, Lithuania, Macedonia, Middle East, Montenegro, The Netherlands, New Zealand, Norway, Philippines, Poland, Portugal, Qatar, Romania, Russia, Saudi Arabia, Serbia, Singapore, Slovakia, Slovenia, Spain, Sweden, Turkey, United Arab Emirates, Ukraine, UK, and USA.

least $50 \%$ of the questions. Table 2 summarizes the characteristics of the remaining respondents $(n=357)$. Two hundred and one female and 152 male physicians completed the survey (four did not indicate their gender). Approximately $75 \%$ of respondents were Europeans, $21 \%$ were from other continents, and $4 \%$ did not specify their country of residence. Most of the respondents worked in academic, state, or teaching hospitals, and $19.6 \%$ were involved in private practice. As expected due to their membership of the ESE, the majority of respondents were endocrinologists (93\%), with half of the remaining participants being gynecologists.

\section{General information about PCOS}

The prevalence of PCOS in participants' countries was estimated as lower than $10 \%$ for $45.6 \%$ of the respondents, between 10 and $20 \%$ for $40.8 \%$ of them and, finally, as higher than $20 \%$ for the remainder. Almost all (98.9\%) replied that, in each patient, they enquired about family history of PCOS, as well as of T2D and cardiovascular disease (CVD). The number of patients with PCOS seen by the respondents in the previous year ranged from $<50$ in $61 \%$ of cases, from 50 to 200 in $34 \%$ of cases, and more than 200 in the remainder. Patient's age at the first visit and diagnosis was $<20$ years for $18.5 \%$ of the respondents, between 20 and 30 years for $76 \%$ of them, and $>30$ years for the remainder.

The source of patients was very heterogeneous and included referrals by dermatologists (8.3\%), an endocrinologist colleague $(6.6 \%)$, gynecologists $(32.3 \%)$, general practitioners $(33.1 \%)$, and by self-referral in $19.7 \%$ of cases. Less than half of the respondents (42.1\%) used a multidisciplinary approach for the management of PCOS; among others, the most frequent collaboration was established with a gynecologist $(86.6 \%)$, a dermatologist (39.1\%), or a nutritionist (35.9\%). There were very few who had collaborated with a psychologist $(6.1 \%)$ or a cardiologist (5.0\%).

\section{Diagnostic criteria of PCOS}

As summarized in Table 3, respondents selected menstrual irregularity as the most common and useful criterion for the diagnosis of PCOS, although high rates were also achieved by hirsutism and biochemical hyperandrogenism. By contrast, ovarian ultrasonography was routinely

Table 3 Frequency in the use of categorical criteria to diagnose PCOS.

\begin{tabular}{|c|c|c|c|c|c|}
\hline \multirow[b]{2}{*}{ Criterion } & \multirow[b]{2}{*}{ Respondents } & \multicolumn{4}{|c|}{ Responses, $n(\%)$} \\
\hline & & Never & Rarely & Sometimes & Often \\
\hline Hirsutism & 351 & $6(1.7)$ & $15(4.3)$ & $83(23.6)$ & $247(70.4)$ \\
\hline Menstrual disturbances & 352 & $3(0.9)$ & $1(0.3)$ & $40(11.4)$ & 308 (87.5) \\
\hline Biochemical hyperandrogenism & 354 & $3(0.8)$ & $14(3.9)$ & $57(16.1)$ & $280(79.1)$ \\
\hline Ovarian ultrasonography & 350 & $10(2.9)$ & $31(8.9)$ & $87(24.9)$ & $222(63.4)$ \\
\hline $\mathrm{AMH}$ & 345 & $196(56.8)$ & $89(25.8)$ & $42(12.2)$ & $18(5.2)$ \\
\hline
\end{tabular}


Table 4 Diagnostic criteria for polycystic ovary syndrome according to the different consensus.

\begin{tabular}{|c|c|c|}
\hline Consensus & Criteria & $\begin{array}{c}\text { Percentage } \\
\quad(\%)\end{array}$ \\
\hline \multirow[t]{2}{*}{$\mathrm{NIH}$} & Hirsutism + menstrual disturbances & 92.9 \\
\hline & $\begin{array}{l}\text { Biochemical hyperandrogenism }+ \\
\text { menstrual disturbances }\end{array}$ & 94.3 \\
\hline \multirow[t]{5}{*}{ Rotterdam } & $\begin{array}{l}\text { Hirsutism + menstrual } \\
\text { disturbances + pelvic US }\end{array}$ & 81.7 \\
\hline & $\begin{array}{l}\text { Biochemical hyperandrogenism }+ \\
\text { menstrual disturbances }+ \\
\text { pelvic US }\end{array}$ & 83.4 \\
\hline & Hirsutism + pelvic US & 0.6 \\
\hline & $\begin{array}{l}\text { Biochemical hyperandrogenism + } \\
\text { pelvic US }\end{array}$ & 0.0 \\
\hline & Menstrual disturbances + pelvic US & 0.9 \\
\hline \multirow[t]{3}{*}{$\begin{array}{l}\text { AE PCOS } \\
\text { Society }\end{array}$} & $\begin{array}{l}\text { Hirsutism or biochemical } \\
\text { hyperandrogenism }+ \text { menstrual } \\
\text { disturbances }\end{array}$ & 11.7 \\
\hline & $\begin{array}{l}\text { Hirsutism or biochemical hyper- } \\
\text { androgenism + pelvic US }\end{array}$ & 0.6 \\
\hline & $\begin{array}{l}\text { Hirsutism or biochemical hyper- } \\
\text { androgenism + menstrual } \\
\text { disturbances + pelvic US }\end{array}$ & 85.7 \\
\hline
\end{tabular}

used by only $60 \%$ of the respondents, mostly in collaboration with a gynecologist. Although consensus statements $(1,2,3,8)$ do not include the anti-Müllerian hormone (AMH) in the diagnostic work-up of PCOS, 17\% of the respondents used it, sometimes or often.

The questions of the ESE survey were not targeted to achieve information on which consensus the respondents based their diagnosis of PCOS, but rather on the choice of each specific criterion. Nonetheless, a reliable perspective was obtained by combining the responses to questions 8-11 (see Table 1 for questions and type of responses). Table 4 summarizes all the combinations of the main criteria used by the respondents. Hyperandrogenism (either hirsutism or biochemical) combined with menstrual abnormalities and, sometimes, with ovarian morphology by ultrasound techniques was selected by the majority of respondents. By contrast, very few selected ovarian morphology combined with either hirsutism or biochemical hyperandrogenism. Collectively, it appears that the NIH criteria (1) are used more frequently than the Rotterdam (2) or the Androgen Excess \& PCOS Society criteria (3). Age, country of residence, speciality, and type of practice did not significantly affect the type of responses. There was a gender difference in the choice of diagnostic criteria used by the respondents, with female doctors preferring the Rotterdam criteria (60.0\%) and male doctors more frequently using NIH criteria (68.3\%; $P=0.01)$; in addition, the two groups differed in age: males, $51.3 \pm 10.3$ years and females, $43.9 \pm 10.4$ years $(P<0.001)$.

\section{Biochemical parameters in the differential diagnosis of hyperandrogenism}

The question about the two most common biochemical indices to be used in the differential diagnosis of hyperandrogenism produced some heterogeneity in the responses, leading to 20 different choices, although mostly including total testosterone or the free androgen index (FAI). It was interesting to note that 75 (21.4\%) of the respondents included direct measurement of free testosterone. In general, respondents chose the combination of total testosterone and 17-hydroxyprogesterone (17OHP) (14\%) or of total testosterone and androstenedione (11\%) to perform the differential diagnosis of hyperandrogenism. The most common biochemical parameters used by the respondents in the differential diagnosis of hyperandrogenism are depicted in Fig. 1.

\section{Long-term concerns about PCOS}

Only one possible concern could be ticked. Obesity and T2D were regarded as the principal long-term concerns for PCOS (64\%). Much less concern was afforded to infertility (20\%). The risk of CVDs was also taken into account by $12 \%$ of respondents, whereas very few respondents selected endometrial cancer (1\%) or psychosocial problems (3\%) as primary concerns (Fig. 4).

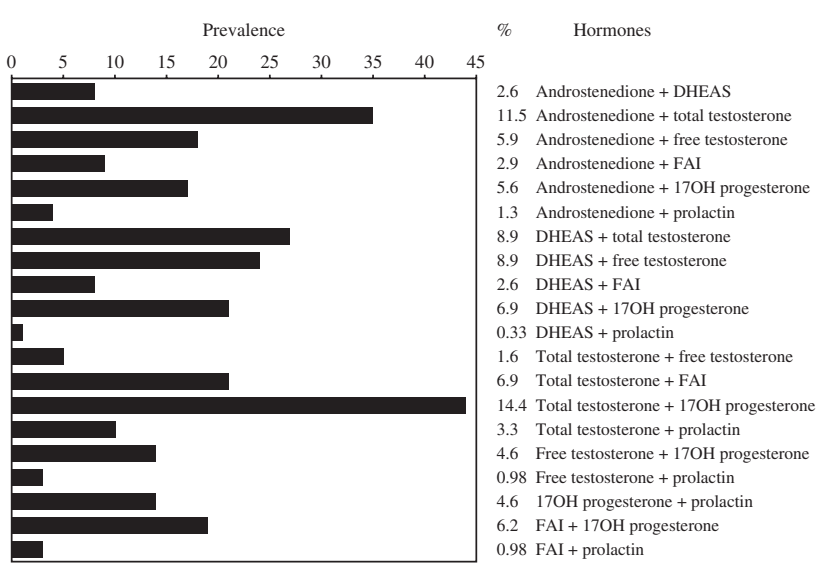

Figure 1

Most common biochemical parameters used in the differential diagnosis of hyperandrogenism. Black bars refer to the absolute number of respondents; the frequency is reported as a number. 


\section{Treatment choices}

The question on the treatment most commonly prescribed for patients with PCOS or, specifically, for fertility problems revealed multiple choices. For the overall treatment of PCOS (Fig. 2A), the respondents' first choice was metformin (33\%), followed by lifestyle modification (25\%), and oral contraceptives (OCs) (22\%). Antiandrogens were prescribed by $13 \%$ and cosmetic procedures for hirsutism by $8 \%$ of the respondents. Metformin and lifestyle intervention were also chosen as a first-line intervention for fertility problems by 36 and $27 \%$ of respondents respectively. More specific treatments of infertility, such as clomiphene citrate (CC) alone or combined with metformin, were prescribed by 9 and $23 \%$, respectively, whereas only $6 \%$ used other methods for induction of ovulation (Fig. 2B). However, most respondents selected multiple treatments for each of the two questions; a list of 27 different combinations was therefore produced (Fig. 3). Intriguingly, 17.8\% of them selected four or five treatments in total.

\section{Discussion}

Worldwide there is an increasing interest of doctors in disorders characterized by androgen excess in women and specifically in PCOS. Historically, PCOS was first described by two gynecologists, Stein \& Leventhal (10), and since menstrual disorders and fertility problems are common features of PCOS, it is not surprising that gynecologists retain a major interest in the subject, as documented by the major diagnostic consensus on PCOS $(1,2,3,9)$. On the other hand, androgen excess per se and related metabolic issues are typical areas of endocrinological interest, and the current survey by ESE clearly expresses the considerable interest of endocrinologists in Europe in the field.

There are some differences between endocrinologists and gynecologists in the definition, diagnosis, and treatment of PCOS, as documented by a recent survey performed in Australia (9) and as shown by many review articles written by gynecologists or endocrinologists (11, $12,13)$. Whereas the latter regarded androgen excess and menstrual irregularity as the major diagnostic criteria, the former were more likely to list ovarian morphology and menstrual irregularities as the main criteria, with androgenization further down the list. Our findings indirectly support the data reported in the Australian study about the approach to PCOS by endocrinologists (9). On the other hand, almost all the respondents selected menstrual disturbances as the major criterion, whereas hirsutism or biochemical hyperandrogenemia was quoted by 94 and 95\% respectively. Interestingly, unlike the Australians, European endocrinologists used ovarian ultrasound quite frequently, which suggests that in Europe there is a good collaboration between the two specialities, additionally supported by the fact that the gynecologists were quoted as the specialists who collaborated most often in the management of patients with PCOS.

Interesting information was obtained on the role of $\mathrm{AMH}$ in the diagnosis of ovarian dysfunction. A significant proportion (17\%) of respondents claimed that they sometimes or often use $\mathrm{AMH}$ measurement as a tool for the diagnosis of ovarian dysfunction in women with PCOS. This could reflect the notion that some authors claimed that $\mathrm{AMH}$ measurement might be a more specific and sensitive surrogate marker to identify polycystic ovarian morphology than the ultrasound techniques (14). In this respect, it is noteworthy that the guidelines for the definition of polycystic ovarian morphology recently published by the Androgen Excess \& PCOS Society conclude that '...although serum AMH generated great expectations as a surrogate marker for the follicle excess, standardization of AMH assays is needed before they can be routinely used for clinical practice and research'(15).

According to the current consensus on diagnostic criteria, most of the respondents selected total testosterone (or FAI) in combination with androstenedione or DHEAS to assess hyperandrogenism. Some of the respondents included the direct measurement of freetestosterone, although there is evidence that common assays are poorly validated and mainly unreliable, especially at the low concentrations characteristic of females $(16,17)$. Others included $17 \mathrm{OHP}$ or prolactin together with testosterone or FAI, with the aim of

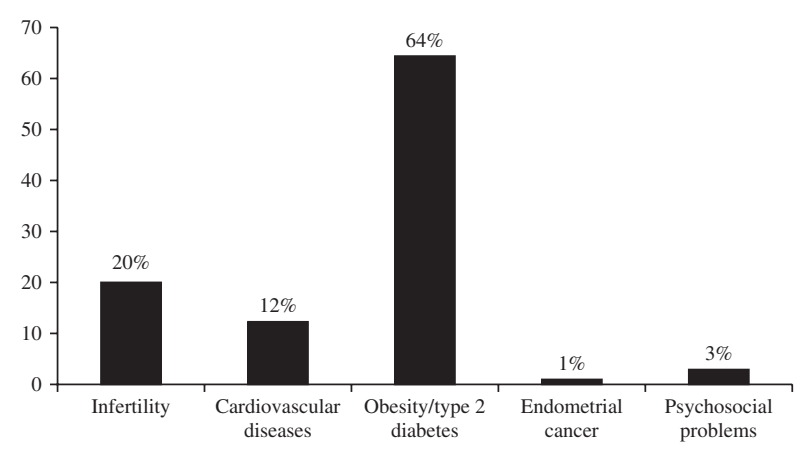

Figure 2

Most important long-term concerns on PCOS by respondents. 

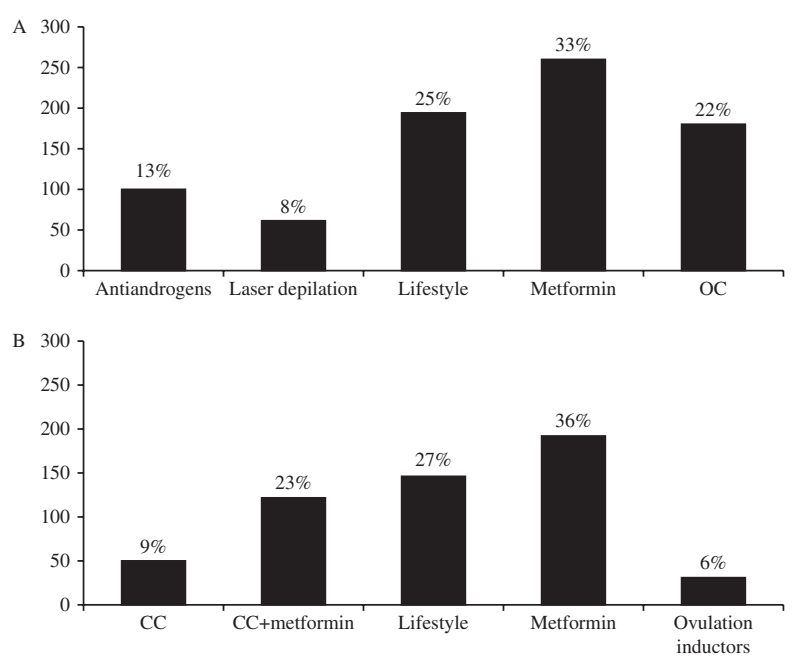

Figure 3

(A) The treatments most commonly prescribed in patients with PCOS, whereas (B) the most common treatments prescribed in PCOS women regarding fertility. Black bars refer to the absolute number of respondents; the frequency is reported as a number.

excluding congenital adrenal hyperplasia and hyperprolactinemic states. These findings could be related to the fact that the question permitted only two possible answers. With respect to androstenedione, women with the more severe phenotype of PCOS are often characterized by elevated levels of both androgens, namely testosterone and androstenedione. In addition, it has been recently shown that serum androstenedione is a more sensitive indicator of PCOS-related androgen excess than serum total testosterone (18). More studies are warranted to corroborate this hypothesis. Measurement of DHEAS, a hormone of predominantly adrenal origin in women, is not routinely required, although when a neoplastic source of androgen excess is suspected a very high DHEAS concentration may be predictive of an androgen-secreting adrenal cancer (19). The evaluation of steroid hormone profiles, by using liquid chromatography combined with tandem mass spectrometry, a recent technique displaying good precision, sensitivity, and high accuracy, would greatly improve the assessment of androgen excess in patients with PCOS $(20,21,22,23)$.

The most important long-term concerns of the majority of respondents were related to metabolic disorders including obesity and $\mathrm{T} 2 \mathrm{D}$ that may represent a specific perspective for endocrinologists. $(5,6)$. The different prevalence of obesity in the clinical series of women with PCOS almost certainly reflects the overall rates of obesity (30-70\%) in different populations $(24,25$, 26). Interestingly, it has been shown that obesity may also cluster in PCOS families (27). Intriguingly, a recent report from the USA provided some evidence of referral bias in the PCOS phenotype, primarily driven by obesity and the severity of disease burden, and that women with PCOS seeking clinical help generally have a more severe PCOS phenotype and are more obese (28). It should be emphasized that abdominal obesity is characterized by a state of relative hyperandrogenemia, due to reduced levels of sex hormone-binding globulin and, consequently, increased bioavailable androgens that are delivered to target tissues. This concept of 'metabolic hyperandrogenemia' merits further investigation from endocrinologists $(29,30)$. Indeed, menstrual disorders are more pronounced when weight gain occurs during puberty rather than during infancy (31). In adult overweight or obese PCOS women, menstrual abnormalities and chronic oligo-anovulation are more frequent, and the degree of hyperandrogenemia tends to be worse than in normal-weight women (29). It is of importance that longitudinal studies have shown that insulin resistance may worsen over time in obese PCOS women (30), and obesity per se is the major risk factor for the early development of T2D $(5,6)$.

Interestingly, the questionnaires show that CVD was considered as the most important long-term

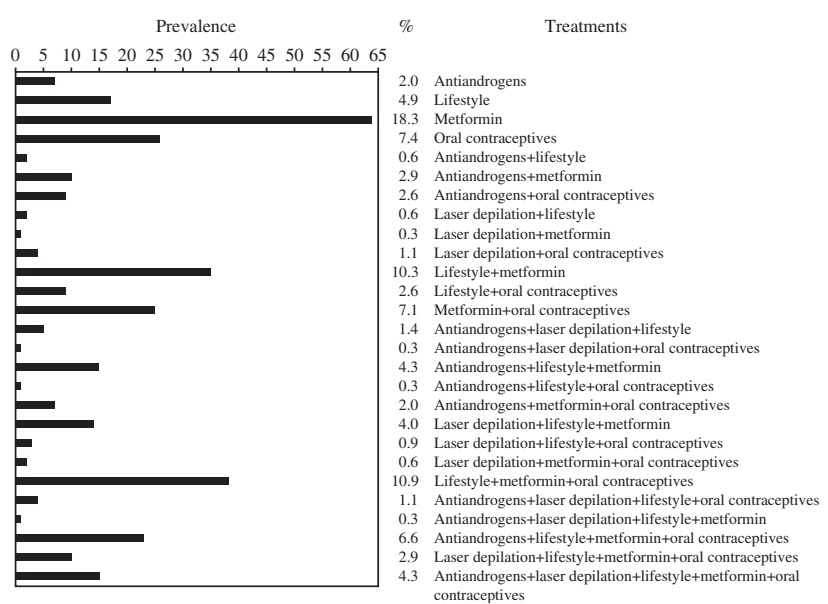

\section{Figure 4}

Prevalence of treatments of PCOS, alone or in combination, according to the question 'Which treatments do you most commonly prescribe for most of your patients with polycystic ovary syndrome?'. Black bars refer to the absolute number of respondents; the frequency is reported as a number. 
concern by only $12 \%$ of the respondents, although an increasing amount of literature in the last 15 years focused on the high prevalence of the metabolic syndrome, other classic and non-classic risk factors for CVD (7), functional and morphological markers of early atherogenesis, and, more recently, on a state of lowgrade inflammation (32), as the pathogenetic mechanisms leading to CVD in women with PCOS. One potential explanation could be that until recently neither cross-sectional nor retrospective studies found an increased prevalence of stroke and myocardial infarction in PCOS (7), although cross-sectional and retrospective studies showed that vascular and coronary atherosclerotic lesions are significantly associated with androgen excess and PCOS status (33). A recent metaanalysis of the available epidemiologic data and a 20-year UK retrospective cohort study have supported the concept that CVDs may be more frequent in patients with PCOS (34). Very few respondents were most concerned about endometrial cancer (1\%), an especially severe complication of chronic endometrial estrogen exposure in untreated oligomenorrheic women with PCOS (7). The close collaboration between endocrinologists and gynecologists that emerged from this survey might represent an opportunity for the former to send their patient to gynecologists not only for ultrasound evaluation of polycystic ovarian morphology and fertility therapeutic intervention, but also for specific prevention or an early diagnosis of gynecological cancers.

Few respondents were concerned about psychosocial problems despite the increasing evidence that psychological symptoms can negatively affect patients with PCOS and may have significant implications for their quality of life. The physical characteristics of PCOS can cause stress and increase the risk for depression (35) and anxiety disorders $(36,37)$. A poorer perception of their quality of life has also been related to a more severe phenotype of women affected by this disorder $(38,39)$. Concerns about body image are associated with fatigue, sleep disturbances, and appetite changes (39). It should be recognised that weight loss may improve depression and quality of life (40). Therefore, besides treating the physical consequences, there is a real need to identify any psychological disorders in women with PCOS and treat them adequately, particularly during critical ages, such as adolescence.

In summary, the ESE survey shows that endocrinologists in Europe consider many of the aspects highlighted in the survey to be relevant for clinical evaluation and management of women with PCOS. In particular, metabolic issues, specifically obesity and insulin resistance, appeared to directly influence the treatment choices. In line with this, most of the respondents selected metformin and lifestyle modification, alone or in combination, as the favorite treatment. It should be argued that, although metformin can improve menstrual irregularity and ovulatory rates in a subset of women with PCOS, there is no evidence that it significantly reduces androgen excess and particularly hirsutism (12). The recent Endocrine Society Clinical Guidelines on the diagnosis and treatment of PCOS (41) have suggested some different approaches in the treatment of PCOS according to specific clinical issues. They recommended combined OCs as first-line management of menstrual abnormalities and hirsutism, if necessary referring to the previous Endocrine Society Clinical Practice Guidelines for the treatment of hirsutism (42). This recommendation was particularly focused on adolescents, although the diagnosis of PCOS at this age still represents a challenging problem and available long-term randomized trials are lacking. For women with PCOS and menstrual irregularities who cannot take or do not tolerate OCs, the guidelines recommended metformin as second-line therapy. Notably, metformin was not recommended for the treatment of hirsutism (and other cutaneous signs of androgen excess). Lifestyle interventional plans are specially recommended, particularly as they favor weight loss, reduce the risk for metabolic comorbidities and, in adolescents, may prevent progression to the classic phenotype of PCOS later in life. Notably, the use of metformin was not necessarily recommended in combination with lifestyle intervention for treatment of obesity $(41,43)$

For the treatment of anovulatory infertility, the guidelines recommended CC, but not metformin; metformin was advised only as an adjuvant therapy to prevent the ovarian hyperstimulation syndrome in women with PCOS undergoing IVF procedures. Finally, the use of other drugs, such as antiandrogens, was not specifically discussed in the guidelines in spite of consistent literature proving their high efficiency in the treatment of symptoms of hyperandrogenism, even alone but especially combined with OCs (12). Undoubtedly, some of the results of this European survey show disagreement with the aforementioned Endocrine Society Guidelines. This may represent a challenge for constructive discussions. At the same time, the survey may form a starting platform for future multicentre prospective trials across Europe and offers an opportunity for an educational effort of the ESE across Europe. 
In conclusion, this ESE survey, although limited by the small sample size mainly composed of endocrinologists as a reflection of the prevalent membership in the ESE, emphasizes some heterogeneity in the management of PCOS by endocrinologists across Europe, and highlights the uncertainties regarding diagnosis and therapeutic approaches to this so common but multifaceted disorder. On the other hand, the questionnaire produced very positive information. It is noteworthy that a substantial majority of ESE endocrinologists use the NIH criteria, which supports the utility of these criteria for the diagnosis and management of PCOS. The younger age of female endocrinonologists with respect to their male counterpart may also suggest that the former might be more willing to adopt the newer Rotterdam criteria (2). In addition, the long-term metabolic risks for obesity and T2D have been well recognized and the popularity of metformin associated or not with lifestyle intervention may reflect the appreciation of these long-term risks by medical endocrinologists. Finally, although there was an inadequate sample of gynecologists/reproductive endocrinologists in this study, the findings support those reported by Cussons et al. (9) that practice patterns differ by specialty, which might support practice-specific diagnostic criteria for PCOS.

\section{Declaration of interest}

The authors declare that there is no conflict of interest that could be perceived as prejudicing the impartiality of the research reported. Bioscientifica Ltd assisted in the administration of the questionnaires but had no role in the analysis and interpretation of the data.

\section{Funding}

The ESE PCOS Special Interest Group has received funds (for meetings and other activities) from the European Society of Endocrinology in the periods 2012-2014. The research activity (the article on PCOS Questionnaires) was part of the activity commissioned by the Society.

\section{References}

1 Zawadzki JK \& Dunaif A. Diagnostic criteria for polycystic ovary syndrome: towards a rationale approach. In Polycystic Ovary Syndrome pp 377-384. Eds A Dunaif, JR Givens, FP Haseltine \& GR Merriam. Boston: Blackwell Scientific Publications, 1992.

2 The Rotterdam ESHRE/ASRM-Sponsored PCOS Consensus Workshop Group. Revised 2003 consensus on diagnostic criteria and long-term health risks related to polycystic ovary syndrome (PCOS). Human Reproduction 200419 41-47. (doi:10.1093/humrep/deh098)

3 Azziz R, Carmina E, Dewailly D, Diamanti-Kandarakis E, EscobarMorreale HF, Futterweit W, Janssen OE, Legro RS, Norman RJ, Taylor AE et al. The Androgen Excess and PCOS Society criteria for the polycystic ovary syndrome: the complete task force report. Fertility and Sterility 200991 456-488. (doi:10.1016/j.fertnstert.2008.06.035)

4 Diamanti-Kandarakis E \& Dunaif A. Insulin resistance and the polycystic ovary syndrome revisited: an update on mechanisms and implications. Endocrine Reviews 201233 981-1030. (doi:10.1210/er. 2011-1034)

5 Pasquali R \& Gambineri A. Glucose intolerance states in women with the polycystic ovary syndrome. Journal of Endocrinological Investigation 201336 648-653. (doi:10.1007/BF03346757)

6 Moran LJ, Misso ML, Wild RA \& Norman RJ. Impaired glucose tolerance, type 2 diabetes and metabolic syndrome in polycystic ovary syndrome: a systematic review and meta-analysis. Human Reproduction Update 201016 347-363. (doi:10.1093/humupd/dmq001)

7 Fauser BC, Tarlatzis BC, Rebar RW, Legro RS, Balen AH, Lobo R, Carmina E, Chang J, Yildiz BO, Laven JS et al. Consensus on women's health aspects of polycystic ovary syndrome (PCOS): the Amsterdam ESHRE/ASRM-Sponsored 3rd PCOS Consensus Workshop Group. Fertility and Sterility 201297 28-38.e25. (doi:10.1016/j.fertnstert.2011. 09.024)

8 Dunaif A \& Fauser BC. Renaming PCOS - a two-state solution. Journal of Clinical Endocrinology and Metabolism 201398 4325-4328. (doi:10.1210/ jc.2013-2040)

9 Cussons AJ, Stuckey BG, Walsh JP, Burke V \& Norman RJ. Polycystic ovarian syndrome: marked differences between endocrinologists and gynaecologists in diagnosis and management. Clinical Endocrinology 200562 289-295. (doi:10.1111/j.1365-2265.2004.02208.x)

10 Stein IF \& Leventhal ML. Amenorrhea associated with bilateral polycystic ovaries. American Journal of Obstetrics and Gynecology 1935 29181.

11 Doll I, Doll R \& Buhling KJ. Diagnosis and therapy of polycystic ovarian syndrome: results of a survey among German gynaecologists with a review on literature. Archives of Gynecology and Obstetrics 2012285 689-697. (doi:10.1007/s00404-011-2014-7)

12 Escobar-Morreale HF, Carmina E, Dewailly D, Gambineri A, Kelestimur F, Moghetti P, Pugeat M, Qiao J, Wijeyaratne CN, Witchel SF et al. Epidemiology, diagnosis and management of hirsutism: a consensus statement by the Androgen Excess and Polycystic Ovary Syndrome Society. Human Reproduction Update 201218 146-170. (doi:10.1093/humupd/dmr042)

13 Sathyapalan T \& Atkin SL. Recent advances in cardiovascular aspects of polycystic ovary syndrome. European Journal of Endocrinology 2012166 575-583. (doi:10.1530/EJE-11-0755)

14 Dewailly D, Gronier H, Poncelet E, Robin G, Leroy M, Pigny P, Duhamel A \& Catteau-Jonard S. Diagnosis of polycystic ovary syndrome (PCOS): revisiting the threshold values of follicle count on ultrasound and of the serum AMH level for the definition of polycystic ovaries. Human Reproduction 201126 3123-3129. (doi:10.1093/ humrep/der297)

15 Dewailly D, Lujan ME, Carmina E, Cedars MI, Laven J, Norman RJ \& Escobar Morreale HF. Definition and significance of polycystic ovarian morphology: a task force report from the Androgen Excess and Polycystic Ovary Syndrome Society. Human Reproduction Update 2014 20 334-352. (doi:10.1093/humupd/dmt061)

16 Rosner W, Auchus RJ, Azziz R, Sluss PM \& Raff H. Position statement: utility, limitations, and pitfalls in measuring testosterone: an Endocrine Society position statement. Journal of Clinical Endocrinology and Metabolism 200792 405-413. (doi:10.1210/jc.2006-1864)

17 Rosner W, Vesper H \& Endocrine Society; and the endorsing organizations. Toward excellence in testosterone testing: a consensus statement. Journal of Clinical Endocrinology and Metabolism 201095 4542-4548. (doi:10.1210/jc.2010-1314)

18 O'Reilly MW, Taylor AE, Crabtree NJ, Hughes BA, Capper F, Crowley RK, Stewart PM, Tomlinson JW \& Arlt W. Hyperandrogenemia predicts metabolic phenotype in polycystic ovary syndrome: the utility of serum androstenedione. Journal of Clinical Endocrinology and Metabolism 201499 1027-1036. 
19 Allolio B \& Fassnacht M. Clinical review: Adrenocortical carcinoma: clinical update. Journal of Clinical Endocrinology and Metabolism 200691 2027-2037. (doi:10.1210/jc.2005-2639)

20 Pugeat M, Déchaud H, Raverot V, Denuzière A, Cohen R, Boudou P \& French Endocrine Society. Recommendations for investigation of hyperandrogenism. Annales d'Endocrinologie 201071 2-7. (doi:10.1016/ j.ando.2009.12.007)

21 Legro RS, Schlaff WD, Diamond MP, Coutifaris C, Casson PR, Brzyski RG, Christman GM, Trussell JC, Krawetz SA, Snyder PJ et al. Total testosterone assays in women with polycystic ovary syndrome: precision and correlation with hirsutism. Journal of Clinical Endocrinology and Metabolism 201095 5305-5313. (doi:10.1210/ jc.2010-1123)

22 Fanelli F, Belluomo I, Di Lallo VD, Cuomo G, De Iasio R, Baccini M, Casadio E, Casetta B, Vicennati V, Gambineri A et al. Serum steroid profiling by isotopic dilution-liquid chromatography-mass spectrometry: comparison with current immunoassays and reference intervals in healthy adults. Steroids 201176 244-253. (doi:10.1016/j. steroids.2010.11.005)

23 Janse F, Eijkemans MJC, Goverde AJ, Lentjes EG, Hoek A, Lambalk CB, Hickey TE, Fauser BC \& Norman RJ. Assessment of androgen concentration in women: liquid chromatography-tandem mass spectrometry and extraction RIA show comparable results. European Journal of Endocrinology 2011165 925-933. (doi:10.1530/EJE-11-0482)

24 Franks S. Polycystic ovary syndrome. New England Journal of Medicine 1995333 853-861. (doi:10.1056/NEJM199509283331307)

25 Ehrmann DA. Polycystic ovary syndrome. New England Journal of Medicine 2005352 1223-1236. (doi:10.1056/NEJMra041536)

26 Yildiz BO, Knochenhauer ES \& Azziz R. Impact of obesity on the risk for polycystic ovary syndrome. Journal of Clinical Endocrinology and Metabolism 200893 162-168. (doi:10.1210/jc.2007-1834)

27 Ehrmann DA, Kasza K, Azziz R, Legro RS, Ghazzi MN \& PCOS/ Troglitazone Study Group. Effects of race and family history of type 2 diabetes on metabolic status of women with polycystic ovary syndrome. Journal of Clinical Endocrinology and Metabolism 200590 66-71. (doi:10.1210/jc.2004-0229)

28 Ezeh U, Yildiz BO \& Azziz R. Referral bias in defining the phenotype and prevalence of obesity in polycystic ovary syndrome. Journal of Clinical Endocrinology and Metabolism 201398 E1088-E1096. (doi:10.1210/jc. 2013-1295)

29 Pasquali R. Obesity and androgens: facts and perspectives. Fertility and Sterility 200685 1319-1340. (doi:10.1016/j.fertnstert.2005.10.054)

30 Borruel S, Fernández-Durán E, Alpañés M, Martí D, Alvarez-Blasco F, Luque-Ramírez M \& Escobar-Morreale HF. Global adiposity and thickness of intraperitoneal and mesenteric adipose tissue depots are increased in women with polycystic ovary syndrome (PCOS). Journal of Clinical Endocrinology and Metabolism 201398 1254-1263. (doi:10.1210/ jc.2012-3698)

31 McCartney CR, Blank SK, Prendergast KA, Chhabra S, Eagleson CA, Helm KD, Yoo R, Chang RJ, Foster CM, Caprio S et al. Obesity and sex steroid changes across puberty: evidence for marked hyperandrogenemia in pre- and early pubertal obese girls. Journal of Clinical Endocrinology and Metabolism 200792 430-436. (doi:10.1210/jc.2006-2002)
32 Repaci A \& Gambineri A. The role of low-grade inflammation in the polycystic ovary syndrome. Molecular and Cellular Endocrinology 2011 335 30-41. (doi:10.1016/j.mce.2010.08.002)

33 Wild RA, Carmina E, Diamanti-Kandarakis E, Dokras A, Escobar-Morreale H, Futterweit W, Lobo R, Norman RJ, Talbott E \& Dumesic DA. Assessment of cardiovascular risk and prevention of cardiovascular disease in women with the polycystic ovary syndrome: a consensus statement by the Androgen Excess and Polycystic Ovary Syndrome (AE-PCOS) Society. Journal of Clinical Endocrinology and Metabolism 201095 2038-2049. (doi:10.1210/jc.2009-2724)

34 de Groot PC, Dekkers OM, Romijn JA, Dieben SW \& Helmerhorst FM. PCOS, coronary heart disease, stroke and the influence of obesity: a systematic review and meta-analysis. Human Reproduction Update 2011 17 495-500. (doi:10.1093/humupd/dmr001)

35 Bishop SC, Basch S \& Futterweit W. Polycystic ovary syndrome, depression and affective disorders. Endocrine Practice 200915 475-482. (doi:10.4158/EP09083.RAR)

36 Jedel E, Waern M, Gustafson D, Landén M, Eriksson E, Holm G, Nilsson L, Lind AK, Janson PO \& Stener-Victorin E. Anxiety and depression symptoms in women with polycystic ovary syndrome compared with controls matched for body mass index. Human Reproduction 201025 450-456. (doi:10.1093/humrep/dep384)

37 Açmaz G, Albayrak E \& İpekMüderris İ. Level of anxiety, depression, self-esteem, social anxiety, and quality of life among the women with polycystic ovary syndrome. Scientific World Journal 20132013851815.

38 Moran LJ, Deeks AA, Gibson-Helm ME \& Teede HJ. Psychological parameters in the reproductive phenotypes of polycystic ovary syndrome. Human Reproduction 201227 2082-2088. (doi:10.1093/ humrep/des114)

39 Livadas S, Chaskou S, Kandaraki AA, Skourletos G, Economou F, Christou M, Boutzios G, Karachalios A, Zerva A, Xyrafis X et al. Anxiety is associated with hormonal and metabolic profile in women with polycystic ovarian syndrome. Clinical Endocrinology 201175 698-703. (doi:10.1111/j.1365-2265.2011.04122.x)

40 Thomson RL, Buckley JD, Lim SS, Noakes M, Clifton PM, Norman RJ \& Brinkworth GD. Lifestyle management improves quality of life and depression in overweight and obese women with polycystic ovary syndrome. Fertility and Sterility 200994 1812-1816. (doi:10.1016/j. fertnstert.2009.11.001)

41 Legro RS, Arslanian SA, Ehrmann DA, Hoeger KM, Murad MH, Pasquali R \& Welt CK. Diagnosis and treatment of polycystic ovary syndrome: an Endocrine Society clinical practice guideline. Journal of Clinical Endocrinology and Metabolism 201398 4565-4592. (doi:10.1210/ jc.2013-2350)

42 Martin KA, Chang RJ, Ehrmann DA, Ibanez L, Lobo RA, Rosenfield RL, Shapiro J, Montori VM \& Swiglo BA. Evaluation and treatment of hirsutism in premenopausal women: an Endocrine Society clinical practice guideline. Journal of Clinical Endocrinology and Metabolism 200893 1105-1120. (doi:10.1210/jc. 2007-2437)

43 Costello M, Shrestha B, Eden J, Sjoblom P \& Johnson N. Insulinsensitising drugs versus the combined oral contraceptive pill for hirsutism, acne and risk of diabetes, cardiovascular disease, and endometrial cancer in polycystic ovary syndrome. Cochrane Database of Systematic Reviews, 2007 CD00555.

Received 28 March 2014

Revised version received 17 July 2014

Accepted 21 July 2014 